\section{Idiopathic Orbital Inflammation Successfully Treated Using Rituximab in a Patient with Rheumatoid Arthritis}

\section{To the Editor:}

Idiopathic orbital inflammation (IOI) is an orbital inflammatory disease, the third most common cause of inflammation within the orbit ${ }^{1}$. Although its etiology is unknown, there is said to be an association with an immune response directed against specific target tissues in the orbit ${ }^{2}$. In many autoimmune diseases, a cellular infiltrate is seen in the affected organ or tissue. Typically, the infiltrate consists of lymphocytes and monocytes, although other immune-related and inflammatory cells can also be seen ${ }^{3}$. We describe a case of IOI within a background of rheumatoid arthritis (RA), and its successful treatment with rituximab (RTX), a chimeric monoclonal antibody against CD20+.

A 43-year-old woman with a 17-year history of seropositive RA presented with a 4-month history of diplopia, right-side proptosis and ptosis, a restriction of movement in the right eye, and a reduction in vision (Figure 1A). RA was very well controlled with adalimumab, an anti-tumor necrosis factor (anti-TNF) biologic agent, which she had been taking fortnightly for 4.5 years, with little early morning stiffness and only the occasional flare involving the joints. She had previously tried a number of diseasemodifying antirheumatic drugs, including methotrexate (MTX), which was stopped following increased hair loss; etanercept, stopped following a transient ischemic attack; sulfasalazine; and hydroxychloroquine. She had no systemic symptoms (fever, weight loss, fatigue, or lethargy), no rashes or dryness of the mouth, no lymphadenopathy or splenomegaly, and no gastrointestinal or cardiorespiratory manifestations. Blood test results did not show any elevated inflammatory markers.

Ocular examination revealed a mild right-side proptosis and persistent choroidal folds affecting the right macula. Visual acuity was reported as 0.6 in the right eye and 0.0 in the left eye, best corrected on logMAR. Confrontational visual field testing showed diplopia on lateral gaze. Optic nerve function was intact with no sign of compression, an absent relative afferent pupillary defect, a normal visual field, and no change in color vision.
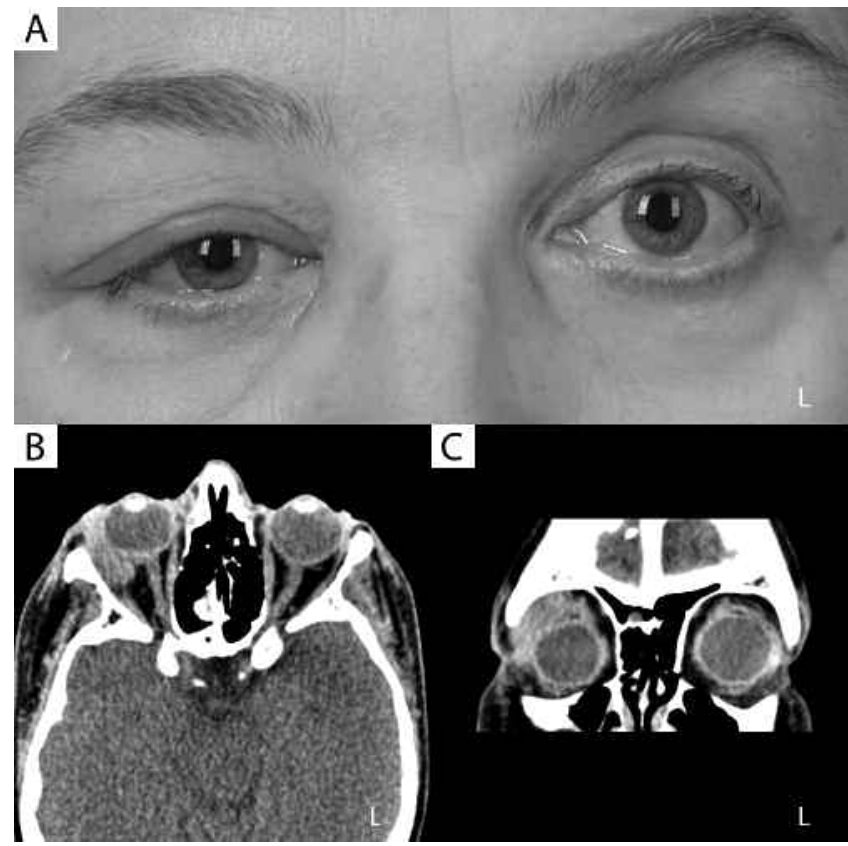

Figure 1. A. Increased swelling of the right eye, with evident conjunctival injection and ptosis. B. and C. Transverse and coronal computed tomography views showing a large orbital inflammatory mass formed predominantly in the posterolateral/lacrimal region of the right orbit.
A computed tomography scan showed a large orbital mass arising from the right lacrimal gland with no bony infiltration (Figure 1B, 1C), and an orbital biopsy led to the diagnosis of IOI. The biopsy revealed a dense mixed cell infiltrate with a predominance of lymphocytes, but with inclusion of a few plasma cells and eosinophils, while immunohistochemistry detected a mixture of B and T cell populations.

Initially, she was treated with 3 pulses of intravenous (IV) methylprednisolone $(250 \mathrm{mg} \times 3)$. In addition, MTX $5 \mathrm{mg} /$ week and folic acid $5 \mathrm{mg} 3$ days/week were started. She was then given RTX infusions $(1000 \mathrm{mg} \times 2)$, administered 2 weeks apart. During the first infusion, she developed some mild allergic symptoms (itching and breathlessness), which settled when the infusion was slowed and with no sign of anaphylaxis. The second infusion 14 days later proceeded without complication and symptoms have since resolved, with a return of her vision. On review a couple of months later, her vision had improved and her ptosis had resolved.

This case is one of the few in the literature that reports successful treatment of IOI using $\mathrm{RTX}^{4,5,6}$

Whether there is a link between IOI and RA remains unknown. A common feature of many autoimmune diseases is the deposition of autoantibody in tissues at various sites around the body, leading to the fixation and activation of complement, and resulting in generation of the inflammatory response $^{7}$. Reports describe development of localized IOI in patients with $\mathrm{RA}^{8,9}$, the first of which describes successful treatment of a 38 -year-old woman with a 6-year history of seropositive RA, with high-dose methylprednisolone ${ }^{9}$. However, despite use of IV methylprednisolone and MTX in our case, symptom resolution is attributed to RTX alone. Indeed, it was only after administration of RTX that there was a reduction of inflammation and return in vision, while the 2 aforementioned treatments proved ineffective.

We question why the ongoing anti-TNF treatment failed to suppress the inflammation within the orbit. Anti-TNF has a role in targeting a specific part of the inflammatory cascade, namely TNF- $\alpha$ or its receptor, or both ${ }^{10}$. The variation of inflammatory mediators is vast, and whether the abundance of one particular mediator corresponds to how sensitive the mass will be to treatment remains unknown. However, in our case, a suggestion that the inflammatory mass may predominantly be formed by CD20+ cells would be supported by the regression of symptoms and inflammation and return in vision following treatment with RTX, a chimeric monoclonal antibody against $\mathrm{CD} 20+$ that is found on the surface of B cells.

Our case highlights the use of RTX for successful treatment of IOI, especially in cases that are refractory to ongoing or newly introduced combinations of immunosuppressant treatments.

IBRAHIM IBRAHIM, Medical Student, The Kellgren Centre for Rheumatology, Manchester Royal Infirmary, Central Manchester University Hospitals NHS Foundation Trust, Manchester M13 9WL; ANNE BARTON, FRCP, PhD, Professor; ABDULLA IBRAHIM, Medical Student, Ninewells Hospital and School of Medicine, University of Dundee, Dundee; PAULINE HO, MRCP, PhD, The Kellgren Centre for Rheumatology, Central Manchester University Hospitals NHS Foundation Trust, Manchester Royal Infirmary, Manchester, UK. Address correspondence to I. Ibrahim;

E-mail: ibrahim.ibrahim@student.manchester.ac.uk

\section{REFERENCES}

1. Lutt JR, Lim LL, Phal PM, Rosenbaum JT. Orbital inflammatory disease. Semin Arthritis Rheum 2008;37:207-22.

2. Shamsi F, Riley F, Chaudhry I, Arat Y. Orbital pseudotumor: Distinct diagnostic features and management. Middle East Afr J Ophthalmol 2008;15:17-27.

3. Jacobs D, Galetta S. Diagnosis and management of orbital pseudotumour. Curr Opin Ophthalmol 2002;13:347-51.

4. Schafranski M. Idiopathic orbital inflammatory disease successfully treated with rituximab. Clin Rheumatol 2009; 28:225-6. 
5. Kurz PA, Suhler EB, Choi D, Rosenbaum JT. Rituximab for treatment of ocular inflammatory disease: A series of four cases. $\mathrm{Br}$ J Ophthalmol 2009;93:546-8.

6. On A, Hirschbein M, Williams H, Karesh J. CyberKnife radiosurgery and rituximab in the successful management of sclerosing idiopathic orbital inflammatory disease. Ophthalm Plast Reconst Surg 2006;22:395-7.

7. Wood P. Understanding immunology (Cell and molecular biology in action). 2nd ed. San Francisco: Benjamin Cummings; 2006.

8. Nabili S, McCarey DW, Browne B, Capell HA. A case of orbital myositis associated with rheumatoid arthritis. Ann Rheum Dis 2002;61:938-9.

9. Panfilio CB, Hernandez-Cossio O, Hernandez-Fustes OJ. Orbital myositis and rheumatoid arthritis: Case report. Arquivos de Neuro-Psiquiatria 2000;58:174-7.

10. Rubin PAD, Foster CS. Etiology and management of idiopathic orbital inflammation. Am J Ophthalmol 2004;138:1041-3.

J Rheumatol 2012;39:7; doi:10.3899/jrheum.111230 
Idiopathic Orbital Inflammation Successfully Treated Using Rituximab in a Patient with Rheumatoid Arthritis

Ibrahim I, Barton A, Ibrahim A, Ho P. Idiopathic orbital inflammation successfully treated using rituximab in a patient with rheumatoid arthritis [letter]. J Rheumatol 2012;39: 1485-6. The affiliation for Prof. Barton should read as follows: ANNE BARTON, FRCP, PhD, Professor, The Kellgren Centre for Rheumatology, Central Manchester University Hospitals NHS Foundation Trust, Manchester Royal Infirmary, Manchester, UK. We regret the error.

doi:10.3899/jrheum.111230.C1 